\title{
Plasmonic Properties of Welded Metal Nanoparticles
}

\author{
Tong Zhang ${ }^{*}{ }^{, 1,3}$, Xiao-Yang Zhang ${ }^{1,2,3}$, Xiao-Jun $\mathrm{Xue}^{3}, \mathrm{Xu}-\mathrm{Feng} \mathrm{Wu}^{1}$, Chen $\mathrm{Li}^{1}$ and A. Hu ${ }^{*}, 2$ \\ ${ }^{1}$ School of Electronic Science and Engineering, Southeast University, Nanjing, 210096, People's Republic of China \\ ${ }^{2}$ Department of Mechanical and Mechatronics Engineering, University of Waterloo, 200 University Avenue West, \\ Waterloo, Ontario N2L 3G1, Canada \\ ${ }^{3}$ Key Laboratory of Micro-Inertial Instrument and Advanced Navigation Technology, Ministry of Education, Nanjing, \\ 210096, People's Republic of China
}

\begin{abstract}
Metal nanostructures show great applications in chemical sensing, biomedical detection, optical-thermal therapy, and optical communications because of their electromagnetic field enhancement properties at the visible and the near-field infrared wavelengths. Such strong optical field enhancement induced by the localized surface plasmon resonance is dependent on the configurations and the sizes of the metal nanoparticles. We presented a numerical investigation of the plasmonic properties of the individually welded silver nanoparticles fabricated by nanojoining technique. It shows that the field enhancement factor in welded silver nanostructures is much larger than in separated silver nanoparticles. The size dependent localized surface plasmon resonance spectra and the polarization sensitivity property of such configurations are also discussed.
\end{abstract}

Keywords: Plasmonics, nanojoining, metal nanoparticles, localized surface plasmon resonance.

\section{INTRODUCTION}

The surface plasmon resonance in metallic nanostructures arises from the collective oscillation of conduction electrons at the surface of metal induced by the incident electromagnetic waves. When the frequency of the movement of the free electrons is approximately the same as the incident light, it leads to the strongest enhancement for the plasmon so called the localized surface plasmon resonance (LSPR). It is one of the major approaches to localize the electromagnetic waves in nanoscale which results in the enormous field enhancement, scattering and absorption of electromagnetic waves. Such unique optical properties open up revolutionary applications in many research fields such as optical microscopy, biochemical sensing and imaging, optical communications, solar-cell designs and targeted therapy [1-7]. For instance, the Raman scattering signals of molecules in the vicinity of the metal nanostructures are on the order of $10^{14}-10^{15}$, leading to the development of the surface-enhanced Raman scattering (SERS) spectroscopy which has been widely used in molecular identification $[8,9]$ and targeting of tumor cells [10]. Another important application of the surface plasmons is the development of nanoscale waveguides and devices. Tight localization and strong enhancement in nanoscale makes the metallic nanostructures promising candidates to develop passive long-chain waveguides [11, 12], activity

*Address correspondence to these authors at the School of Electronic Science and Engineering, Southeast University, Nanjing, 210096, People's Republic of China; Tel: 0086-25-83792449-833; Fax: 0086-25-83363222; E-mail: tzhang@seu.edu.cn

Department of Mechanical and Mechatronics Engineering, University of Waterloo, 200 University Avenue West, Waterloo, Ontario N2L 3G1, Canada; Tel: 1-519-8884567-35464; Fax: 1-519-8855862;

E-mail: a2hu@uwaterloo.ca plasmonic lasers $[13,14]$ and tunable optical filters [15] for the next generation high-density integration. It is also the current interest to study the single-molecule fluorescence [16] and the high-order nonlinear optical response [14, 17] using the metallic nanostructures because of the strong nearfield enhancement arisen from the interaction of the excitedstate optical signals with the local electric fields of the metallic nanoparticles.

Many efforts have been devoted to the fabrication of metallic nanostructures by using chemical synthesis. Among the various metallic materials, silver and gold are widely investigated because the plasmon frequencies of these noble metals are in the visible region and can be extended to the near-infrared region. As the resonance frequencies and the field enhancement factors are dependent on several factors, for example, the shapes and the sizes of the nanoparticles, the interaction between the adjacent nanoparticles and the influence of the surrounded dielectrics, the fabrication techniques of the metallic nanostructures is of considerable interest for plasmonics. Varieties of silver and gold nanostructures with different shapes and sizes have been synthesized chemically and studied systematically in previous [2, 18-23]. Generally speaking, the plasmon resonance peaks of the anisotropic structures such as nanoplates and nanorods shift towards longer wavelengths compared with the spherical nanoparticles [2]. The LSPR spectra of anisotropically etched nanoparticles such as nanoflowers, nanostars and nanopyramids show multiresonance peaks or broad bandwidths [18-23]. In additional, complex nanostructures such as aggregative nanoparticles with polymer cladding, core-shell nanospheres and dimers have also been synthesized for the development of high sensitive SERS probes [10, 24-31]. The field enhancement factors of such complex nanostructures can be increased 
significantly compared with the individual metallic nanoparticle.

Compared with chemical synthesis methods, nanolithography and nanoimprint show advantages in the production of complex planar metallic nanostructures and ordered arrays of plasmonic structures [4]. These techniques offer a scalable approach to construct large-scale complex nanostructures with unique optical properties. Ordered nanoparticle array $[32,33]$ and nanopin resonator array [34] with tunable LSPR spectra have been demonstrated. The optical properties of such plasmonic arrays can be tuned accurately by changing the gap distances and the shapes of the adjacent nanonparticles. Nanolithography is also used to develop plasmonic structures supporting Fano resonances $[35,36]$.

The chemical anisotropically etching and nanolithography have been thoroughly investigated to construct complex metallic nanostructures. There is, however, only a few pioneer works on the nanojoining for the development of metallic building blocks in nanoscale [6, 37-44]. Nanojoining here is referred to the welding and sintering approaches to construct permanent unions or connections between nanoscaled building blocks by physical or chemical triggering. Such techniques have been shown as the critical methods to fabricate nanoscaled single electron transistor, metal-semiconductor junctions, and functional circuits using nanowires, nanotubes and nanoparticles [38, 39]. Recently, it has been demonstrated that the adjacent metallic nanoparticles can be jointed permanently by laser welding $[6,37,40]$ and sintering triggered thermally [41, 42] or chemically $[43,44]$. It provides a new strategy to fabricate large-scale metallic nanostructures with interesting plasmonic properties. In this paper, we investigated the plasmonic properties of welded silver nanospheres using three-dimensional (3D) finite element method (FEM). We displayed the near-field intensity enhancement and the movement of the LSPR peaks of the welded nanoparticles. Compared simulation results showed that nanojoining is a simple approach to achieve nanostructures with tunable LSPR spectral and polarization-sensitivity properties in longwavelength range.

\section{NUMERICAL MODELING}

$3 \mathrm{D}$ models based on the numerical methods such as the discrete dipole approximation (DDA), the finite difference time domain (FDTD) method, and FEM have been used to simulate the near-field intensity distribution of metallic nanostructures with arbitrary geometries by solving the Maxwell's equations numerically [45-48]. Compared with other numerical methods, 3D-FEM shows high accuracy for modeling irregular structures and simulating fine elements within large domains. In the algorithm, non-uniform meshes are efficient to produce convergence and save the computing resources. Our results are obtained from COMSOL Multiphysics, a numerical simulation package based on 3DFEM which has been widely used in the simulation of plasmonic devices [48-50]. Khoury et al. have demonstrated the validity and the accuracy for plasmonic-nanostructure modeling using COMSOL Multiphysics [48].
In our model, silver nanospheres were chosen as the basic elements for nanojoing because the optical loss of silver is much lower compared with other noble metals in the visible range. It should be noted that the simulation results including the intensity distributions and the peak position of the LSPR spectra are also dependent on the data and the fitting methods of the dielectric constant of metals [51]. However, there are still some controversies of which experimental data are more suitable and accurate for the numerical model [51-53]. In our model, the complex permittivity of the silver is analytically fitted by DrudeLorentz models [54] using experimental data from [55].

The geometries of the silver nanostructures we considered are illustrated in Fig. (1). Fig. (1a, b) show the cross section views ( $x-y$ plane) of two adjacent nanospheres and two welded nanospheres, respectively. The radius of the nanosphere is $R$ and the gap distance between the two nanospheres is $D$. Fig. (1c, d) shows the Transmission electron microscopy (TEM) images of the silver nanostructures achieved in our experiment. TEM samples were prepared by dripping irradiated Ag nanoparticles solution on carbon films coated $\mathrm{Cu}$ grids. The TEM observation was gotten by the JEOL JEM-2010F device with the voltage of $200 \mathrm{kV}$ and the current of $100 \mu \mathrm{A}$. Silver dimers with a positive gap distance (shown in Fig.1c) can be obtained easily by chemical synthesis [28, 29]. By using femtosecond laser radiation [6], the two adjacent silver nanospheres can be welded permanently with a negative gap distance (as shown in Fig. 1d). In our numerical model, the silver nanostructures were surrounded by air with a refractive index $n=1$. The shell surrounded the silver nanoparticles as shown in Fig. (1c, d) is the dielectric layer which has been identified in our previous work [42]. Because the thickness of the shell is only about $2 \mathrm{~nm}$ or thinner, we ignored this layer in our model. The input and output boundaries were set to be the port condition. The rectangular wave with a transverse magnetic (TM) 01 mode is used as the incident light. Scattering boundary condition is used at other boundaries.

\section{RESULTS AND DISCUSSION}

Fig. (2) shows the cross-sectional views of the normalized electric field $|E|$ distributed at the surface of the silver dimer and the welded spheres at resonance. Fig. (2a, b) show the electric field distribution at the center of the nanostructures at the $x-y$ plane as illustrated in Fig. $(\mathbf{1 a}, \mathbf{b})$. Fig. (2c, d) show the electric field distribution at the neck position of the dimer and the welded nanospheres from the view of $z-x$ plane, respectively. One can see clearly the distinct intensity distributions at the junction of the nanospheres before and after the nanospheres are welded. For silver dimer with a positive gap distance, the near-field intensity distributes mainly in the center of the gap region between the nanospheres. In comparison, for welded nanospheres, the intensity mainly distributes in the sharp corner region like a bright ring. The field distribution of the welded nanospheres is exceptional and different from that of other anisotropic structures such as nanoplates and nanorods which the intensity mainly distributes at their edges. 

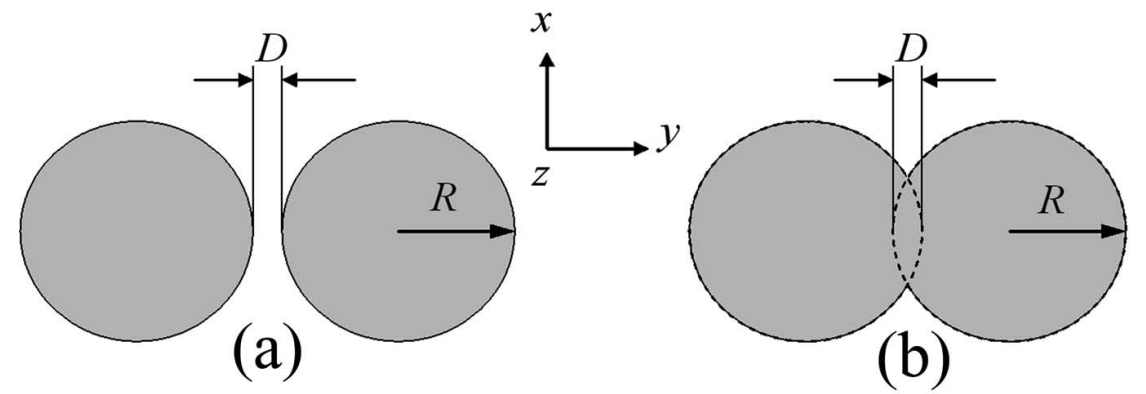

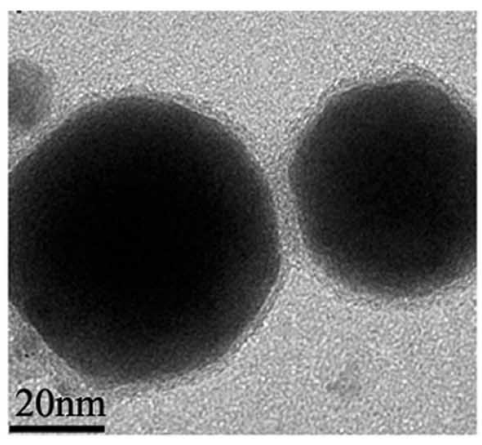

(c)

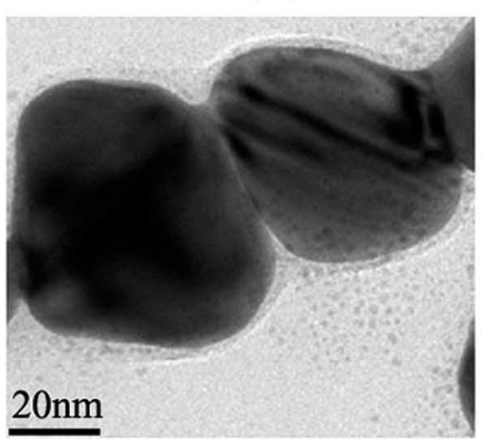

(d)

Fig. (1). The geometries of the adjacent silver nanospheres and welded nanospheres are shown in (a) and (b), respectively. The TEM images of the silver nanospheres synthesized chemically before and after the laser radiation are shown in (c) and (d), respectively.
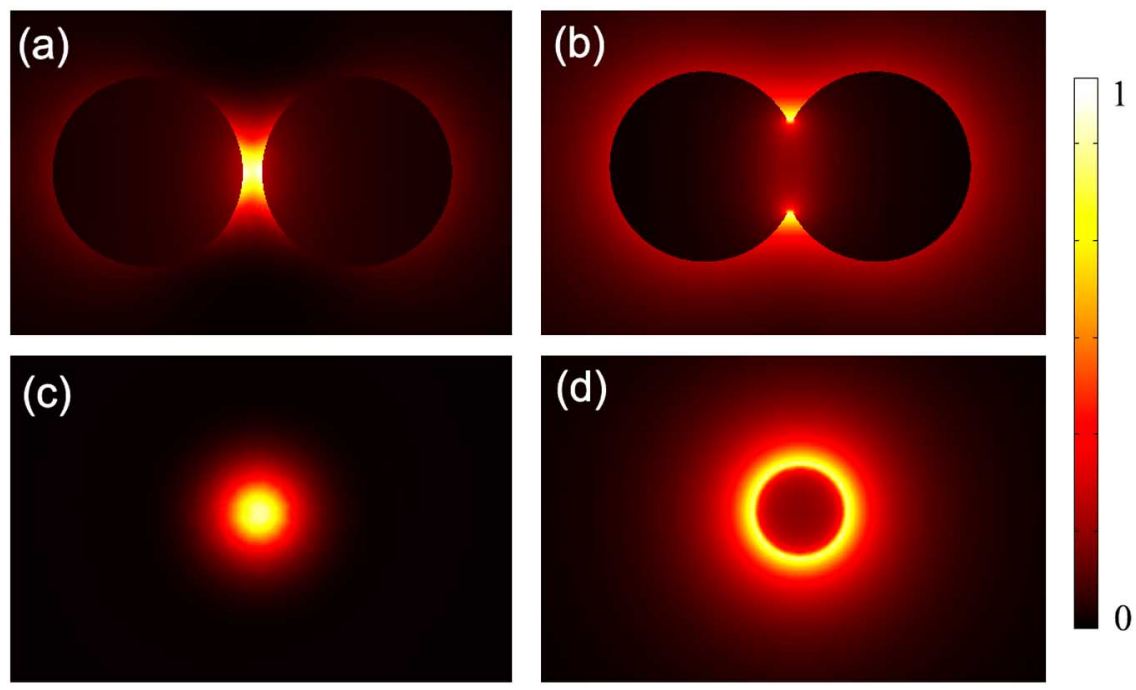

Fig. (2). The cross-sectional views of the normalized electric field $|E|$ distributed at the surface of the nanostructures. (a) and (b) show the electric field distribution at the center of the silver dimer and the welded spheres at resonance from the view of $x-y$ plane. (c) and (d) show the electric field distribution at the neck position of the dimer and the welded nanospheres from the view of $z$ - $x$ plane. The wavelength of the incident light is $415 \mathrm{~nm}$ in (a) and (c), and $619 \mathrm{~nm}$ in (b) and (d).

Fig. (3) shows the comparison of the normalized electric fields as a function of the wavelength for different nanostructures. Compared with an individual nanosphere (the red curve), the amplitudes of the electric field at resonance can be enhanced by three orders of magnitude when two nanospheres are closely adjacent or welded permanently. A smaller peak at about $370 \mathrm{~nm}$ is observed for both silver dimers and welded spheres which may correspond to the quadrupole resonance. For silver dimers, the peak at the longitude plasmon resonance (longwavelength resonance) shows slightly red-shift phenomenon when the distance of the two adjacent nanospheres is decreased from $5 \mathrm{~nm}$ to $0.5 \mathrm{~nm}$. Two main resonance peaks corresponding to the transverse resonance (at the wavelength of $420 \mathrm{~nm}$ ) and the longitude resonance (at the wavelength of $540 \mathrm{~nm}$ ) are observed with $D=0.5 \mathrm{~nm}$. When the two nanospheres are welded with a nearly zero distance $(D=$ $0.5 \mathrm{~nm}$ ), the longitude resonance peak shows a significant red-shift phenomenon up to $1080 \mathrm{~nm}$. However, the amplitude of the peak is lower than that of the transverse resonance at about $470 \mathrm{~nm}$. By increasing the negative distance between the two nanospheres from $-0.5 \mathrm{~nm}$ to $-5 \mathrm{~nm}$, 
these plasmon resonance peaks show blue-shift phenomenon and the amplitude of the short-wavelength resonance peaks decreases significantly. For the most common geometries of the welded spheres obtained in our experiment $(D$ is about $5 \mathrm{~nm}$ ), the primary resonance is the longitudinal plasmon resonance at the wavelength range between $600 \mathrm{~nm}$ and $700 \mathrm{~nm}$. Such strong electromagnetic field enhancement at the long wavelength makes the welded silver nanospheres very useful for the development of SERS probes for single molecular detecting and imaging.

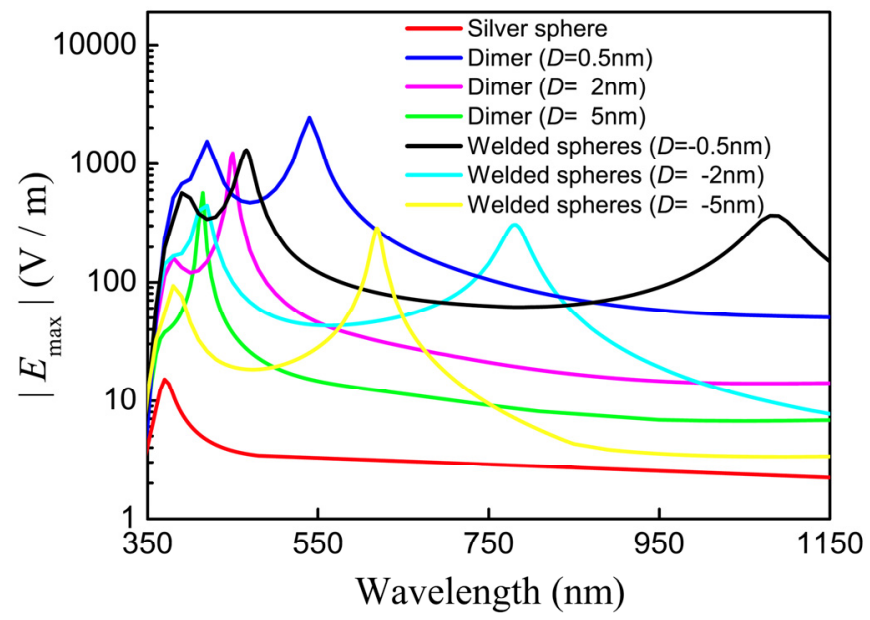

Fig. (3). The comparison of the LSPR spectra as a function of the wavelength for individual silver nanosphere, two adjacent silver nanospheres with $D=0.5 \mathrm{~nm}, 2 \mathrm{~nm}$ and $5 \mathrm{~nm}$ and two welded silver nanospheres with $D=-0.5 \mathrm{~nm}, 2 \mathrm{~nm}$ and $-5 \mathrm{~nm}$. The radius of the nanosphere is $25 \mathrm{~nm}$.

Meantime, we found that the full width at half maximum (FWHM) of the LSPR spectra is dependent on the maximum amplitude of the resonance peak. For separated silver nanosphere, the FWHM of the LSPR spectrum is much wider than that of the other nanostructures because the maximum amplitude of the resonance peak of the separated nanosphere is only about $15 \mathrm{~V} / \mathrm{m}$. In comparison, the FWHM of the main peak for welded nanospheres with $D=-$ 0.5 becomes much narrower. It is only $1 / 3$ of that of the separated silver nanosphere. Therefore, we believe that nanojoining is an effective method to create welded metallic nanostrucutres with shape resonance peaks of the LSPR spectra.

Fig. (4) shows the size-dependent near-field electric field distribution as a function of the wavelength for the welded nanospheres. We set a fiducial radius $R_{0}=25 \mathrm{~nm}$. By enlarging or reducing the volume of the welded nanospheres proportionally, the position and the amplitude of the primary resonance peak are changed significantly. It shows that the resonance peak moves red-shiftily in a wide range when the radius $R$ of the nanospheres increases. The maximum value of the amplitude of the electric field at resonance is obtained when the radius of the nanospheres is about $25 \mathrm{~nm}$. For welded nanospheres with a large radius $(R=50 \mathrm{~nm})$, the electric-field enhancement at the long wavelengths is no longer obvious. This result indicates that choosing silver nanoparticles with appropriate sizes is critical to achieve the strongest field enhancement for welded nanostructures.

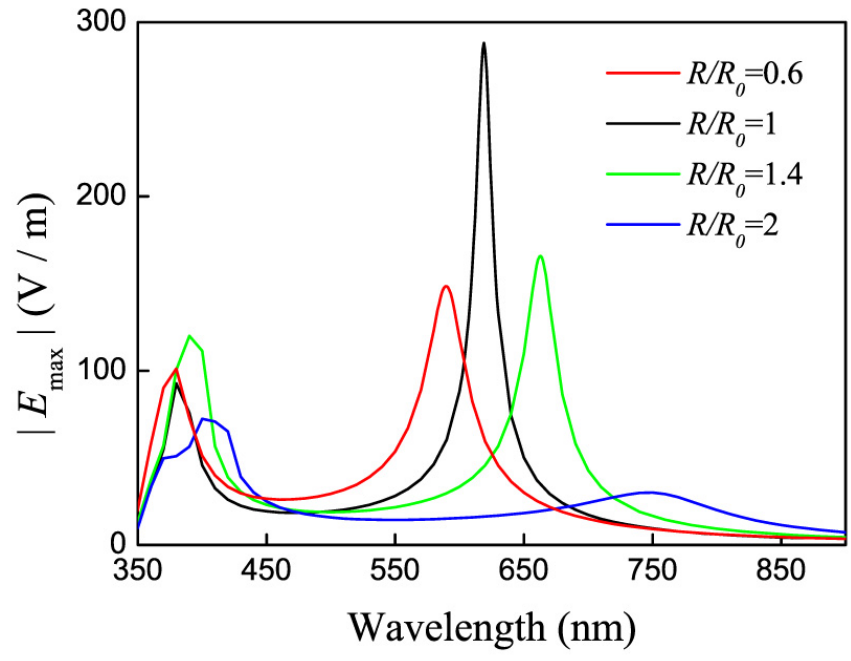

Fig. (4). The size-dependent LSPR spectra as a function of the wavelength for the welded nanospheres. $R_{0}=25 \mathrm{~nm}$ which is a fiducial radius.

We further investigated the angular dependent near-field electric-field distribution of the welded nanospheres as shown in Fig. (5). As shown, the excitation of the LSPR is highly dependent on the angle $\theta$ between the direction of the electric-field of the incident light and the longitudinal direction of the welded nanospheres ( $x-y$ plane). Fig. (5a-d) show the cross-sectional views of the normalized electric field $|E|$ distributed at the surface of the welded nanospheres with different $\theta$ at resonance. When $\theta=0^{\circ}$, the electromagnetic wave gets resonance and mainly distributes at the corner region. The electric-field distribution changes in according to the rotation angle of the nanostructure. When $\theta$ $=90^{\circ}$, the electromagnetic wave distributes mainly at the surface of the nanospheres, no longer at the corner region. Fig. (5e) shows the LSPR spectra as a function of $\theta$. By rotating the welded nanospheres counterclockwise from $0^{\circ}$ to $90^{\circ}$, the maximum value of $|E|$ at resonance decreases significantly. When $\theta=90^{\circ}$, the resonance peak at the long wavelength disappear. Fig. (5f) shows the maximum value of the amplitude at the resonance wavelength of $619 \mathrm{~nm}$ as a function of $\theta$. Such polarization sensitive property of the welded nanospheres is mainly induced by the anisotropic surface plasmon excitation nature of metallic nanostructures and can be used to control the resonant intensity of the nanostructures. It is useful in the development of nanoscaled SERS probes and nanoscaled optical switches.

\section{CONCLUSION}

In this paper, we investigated the plasmonic properties for the silver nanostructures fabricated using nanojoining. We showed that nanojoining is a simple technique to develop welded metallic nanostructures with unique LSPR spectra and polarization-sensitivity properties different from the conventional chemically synthesized metallic nanostructures. We showed that the LSPR spectra of such welded nanospheres can be tuned in a wide range and the field enhancement factor in welded silver nanostructures is much larger than in separated silver nanoparticles. Such welded silver nanostructures are useful for the development of high sensitive SERS probe and other nanoscaled photonic devices. 

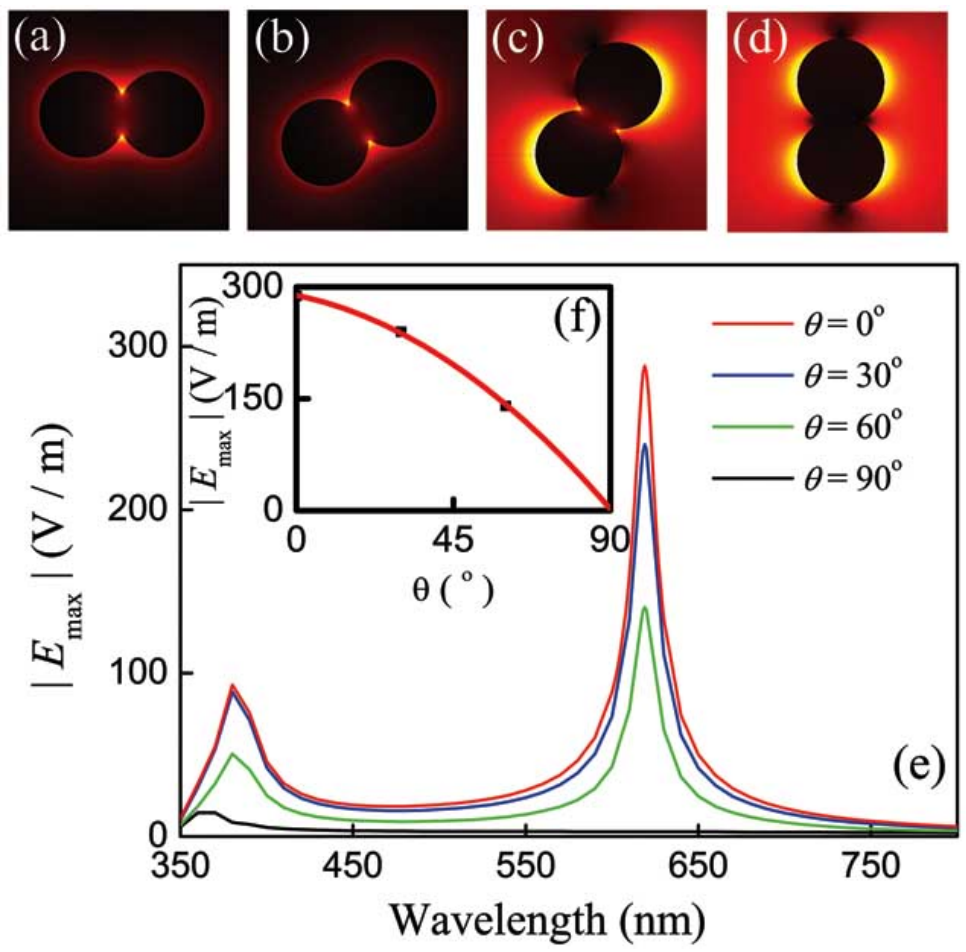

Fig. (5). The angular dependent near-field electric-field distribution of the welded nanospheres with $\theta=0^{\circ}$ in $(\mathbf{a}), \theta=30^{\circ}$ in $(\mathbf{b}), \theta=60^{\circ}$ in (c) and $\theta=90^{\circ}$ in (d). The LSPR spectra as a function of $\theta$ are shown in (e). Maximum value of the amplitude at the resonance wavelength of $619 \mathrm{~nm}$ as a function of $\theta$ is shown in (f).

\section{ACKNOWLEDGEMENTS}

This work is supported by NSFC No. 60977038, NSFCResearch Fund for International Young Scientists No.60910187, the Scientific Research Foundation of Graduate School of Southeast University No. YBJJ0925, Graduate Innovation Program of Jiangsu Province No. CX09B_050Z, and the Foundation of Key Laboratory of Micro-Inertial Instrument and Advanced Navigation Technology, Ministry of Education, China.

\section{REFERENCES}

[1] Jain PK, Huang XH, El-Sayed IH, El-Sayed MA. Noble metals on the nanoscale: Optical and photothermal properties and some applications in imaging, sensing, biology, and medicine. Acc Chem Res 2008; 4: 1578-86.

[2] Lu XM, Rycenga M, Skrabalak SE, Wiley B, Xia YN. Chemical synthesis of novel plasmonic nanoparticles. Annu Rev Phys Chem 2009; 60: 167-92.

[3] Yonzon CR, Stuart DA, Zhang XY, McFarland AD, Haynes CL, Van Duyne RP. Towards advanced chemical and biological nanosensors - An overview. Talanta 2005; 67: 438-48.

[4] Henzie J, Lee J, Lee MH, Hasan W, Odom TW. Nanofabrication of plasmonic structures. Annu Rev Phys Chem 2009; 60: 147-65.

[5] Stewart ME, Anderton CR, Thompson LB, et al. Nanostructured plasmonic sensors. Chem Rev 2008; 108: 494-521.

[6] Hu A, Panda SK, Khan MI, Zhou Y. Laser welding, microwelding, nanowelding and nanoprocessing. Chin J Lasers 2009; 36: 3149-59.

[7] Atwater HA, Polman A. Plasmonics for improved photovoltaic devices. Nat Mater 2010; 9; 205-13.

[8] Hu A, Lu QB, Duley WW, Rybachuk M. Spectroscopic characterization of carbon chains in nanostructured tetrahedral carbon films synthesized by femtosecond pulsed laser deposition. J Chem Phys 2007; 126: 154705.

[9] Nie SM, Emory SR. Probing single molecules and single nanoparticles by surface-enhanced Raman scattering. Science 1997; 275: 1102-6.

[10] Yang J, Wang ZY, Tan XB, et al. A straightforward route to the synthesis of a surface-enhanced Raman scattering probe for targeting transferrin receptor-overexpressed cells. Nanotechnol 2010; $21: 345101$.

[11] de Waele R, Koenderink AF, Polman A. Tunable nanoscale localization of energy on plasmon particle arrays. Nano Lett 2007; 7: 2004-8.

[12] Koenderink AF. Plasmon nanoparticle array waveguides for single photon and single plasmon sources. Nano Lett 2009; 9: 4228-33.

[13] Noginov MA, Zhu G, Belgrave AM, et al. Demonstration of a spaser-based nanolaser. Nature 2009; 460: 1110-3.

[14] Kim S, Jin JH, Kim Y-J, Park I-Y, Kim Y, Kim SW. Highharmonic generation by resonant plasmon field enhancement. Nature 2008; 453: 757-60.

[15] Su XR, Zhang ZS, Zhang LH, et al. Plasmonic interferences and optical modulations in dark-bright-dark plasmon resonators. Appl Phys Lett 2010; 96: 043113.

[16] Zhang J, Fu Y, Chowdhury MH, Lakowicz JR. Metal-Enhanced single-molecule fluorescence on silver particle monomer and dimer: Coupling effect between metal particles. Nano Lett 2007; 7: 2101-7.

[17] Jin RC, Jureller JE, Kim HY, Scherer NF. Correlating second harmonic optical responses of single $\mathrm{Ag}$ nanoparticles with morphology. J Am Chem Soc 2005; 127: 12482-3.

[18] Liang HY, Li ZP, Wang WZ, Wu YS, Xu HX. Highly surfaceroughened "flower-like" silver nanoparticles for extremely sensitive substrates of surface-enhanced Raman scattering. Adv Mater 2009; 21: 4614-8.

[19] Lu L, Kobayashi A, Tawa K, Ozaki Y. Silver nanoplates with special shapes: Controlled synthesis and their surface plasmon resonance and surface-enhanced Raman scattering properties. Chem Mater 2006; 18: 4894-901.

[20] Mulvihill MJ, Ling XY, Henzie J, Yang PD. Anisotropic etching of silver nanoparticles for plasmonic structures capable of singleparticle SERS. J Am Chem Soc 2010; 132: 268-74.

[21] Cobley CM, Rycenga M, Zhou F, Li ZY, Xia YN. Etching and growth: An intertwined pathway to silver nanocrystals with exotic shapes. Angew Chem Int Ed 2009; 48: 4824-7.

[22] Naumov II, Li ZY, Bratkovsky AM. Plasmonic resonances and hot spots in Ag octopods. Appl Phys Lett 2010; 96: 033105.

[23] Stoerzinger KA, Hasan W, Lin JY, Robles A, Odom TW Screening nanopyramid assemblies to optimize surface enhanced Raman scattering. J Phys Chem Lett 2010; 1: 1046-50. 
[24] Feng JJ, Gernert U, Sezer M, et al. Novel Au-Ag hybrid device for electrochemical $\mathrm{SE}(\mathrm{R}) \mathrm{R}$ spectroscopy in a wide potential and spectral range. Nano Lett 2009; 9: 298-303.

[25] Prodan E, Radloff C, Halas NJ, Nordlander P. A hybridization model for the plasmon response of complex nanostructures. Science 2003; 302: 419-22.

[26] Li JF, Huang YF, Ding Y, et al. Shell-isolated nanoparticleenhanced Raman spectroscopy. Nature 2010; 464: 392-5.

[27] Yang MX, Chen T, Lau WS, et al. Development of polymerencapsulated metal nanoparticles as surface-enhanced Raman scattering probes. Small 2009; 5: 198-202.

[28] Chen HJ, Sun ZH, Ni WH, et al. Plasmon coupling in clusters composed of two-dimensionally ordered gold nanocubes. Small 2009; 5: 2111-9.

[29] Li WY, Camargo PHC, Lu XM, Xia YN. Dimers of silver nanospheres: Facile synthesis and their use as hot spots for surfaceenhanced Raman scattering. Nano Lett 2009; 9: 485-90.

[30] Chen T, Wang H, Chen G, et al. Hotspot-induced transformation of surface-enhanced Raman scattering fingerprints. ACS Nano 2010; 4: 3087-94.

[31] He D, Hu B, Yao QF, Wang K, Yu SH. Large-scale synthesis of flexible free-standing SERS substrates with high sensitivity: Electrospun PVA nanofibers embedded with controlled alignment of silver nanoparticles. ACS Nano 2009; 3: 3993-4002.

[32] Jensen TR, Malinsky MD, Haynes CL, Van Duyne RP. Nanosphere lithography: Tunable localized surface plasmon resonance spectra of silver nanoparticles. J Phys Chem B 2000; 104: 10549-56.

[33] Berkovitch N, Ginzburg P, Orenstein M. Concave plasmonic particles: Broad-band geometrical tunability in the near-Infrared. Nano Lett 2010; 10: 1405-8.

[34] Wang S, Pile DFP, Sun C, Zhang X. Nanopin plasmonic resonator array and its optical properties. Nano Lett 2007; 7: 1076-80.

[35] Gopinath A, Boriskina SV, Premasiri WR, Ziegler L, Reinhard BM, Dal Negro L. Plasmonic nanogalaxies: Multiscale aperiodic arrays for surface-enhanced Raman sensing. Nano Lett 2009; 9: $3922-9$.

[36] Lassiter JB, Sobhani H, Fan JA, et al. Fano resonances in plasmonic nanoclusters: Geometrical and chemical tunability. Nano Lett 2010; 10: 3184-9.

[37] Zhou N, Hu AM. Nanojoining - An integration technology for nanodevices and nanosystems. Nanotechnol (unpublished).

[38] Hu JT, Ouyang M, Yang PD, Lieber CM. Controlled growth and electrical properties of heterojunctions of carbon nanotubes and silicon nanowires. Nature 1999; 399: 48-51.

[39] Cui QZ, Gao F, Mukherjee S, Gu ZY. Joining and interconnect formation of nanowires and carbon nanotubes for nanoelectronics and nanosystems. Small 2009; 5: 1246-57.

[40] Tanaka Y, Yoshikawa H, Itoh T, Ishikawa M. Laser-induced selfassembly of silver nanoparticles via plasmonic interactions. Opt Express 2009; 17: 18760-7.
[41] Ide E, Angata S, Hirose A, Kobayashi KF. Metal-metal bonding process using Ag metallo-organic nanoparticles. Acta Mater 2005; 53: $2385-93$

[42] $\mathrm{Hu} \mathrm{A}$, Guo JY, Alarifi $\mathrm{H}$, et al. Low temperature sintering of $\mathrm{Ag}$ nanoparticles for flexible electronics packaging. Appl Phys Lett 2010; 97: 153117

[43] Lee Y, Choi JR, Lee KJ, Stott NE, Kim D. Large-scale synthesis of copper nanoparticles by chemically controlled reduction for applications of inkjet-printed electronics. Nanotechnology 2008; 19: 415604.

[44] Magdassi S, Grouchko M, Berezin O, Kamyshny A. Triggering the sintering of silver nanoparticles at room temperature. ACS Nano 2010; 4: 1943-8.

[45] Zhao J, Pinchuk AO, Mcmahon JM, et al. Methods for describing the electromagnetic properties of silver and gold nanoparticles. Acc Chem Res 2008; 41: 1710-20.

[46] Perassi EM, Hernandez-Garrido JC, Moreno MS, Encina ER, Coronado EA, Midgley PA. Using highly accurate 3D nanometrology to model the optical properties of highly irregular nanoparticles: A powerful tool for rational design of plasmonic devices. Nano Lett 2010; 10: 2097-104.

[47] Futamata M, Maruyama Y, Ishikawa M. Local electric field and scattering cross section of $\mathrm{Ag}$ nanoparticles under surface plasmon resonance by finite difference time domain method. J Phys Chem B 2003; 107: 7607-17.

[48] Khoury CG, Norton SJ, Vo-Dinh T. Plasmonics of 3-D nanoshell dimers using multipole expansion and finite element method. ACS Nano 2009; 3: 2776-88.

[49] Zhang X-Y, Hu A, Wen JZ, et al. Numerical analysis of deep subwavelength integrated plasmonic devices based on SemiconductorInsulator-Metal strip waveguides. Opt Express 2010; 18: 18945-59.

[50] Zhang X-Y, Hu A, Zhang T, Xue X-J, Wen JZ, Duley WW. Subwavelength plasmonic waveguides based on $\mathrm{ZnO}$ nanowires and nanotubes: A theoretical study of thermo-optical properties. Appl Phys Lett 2010; 96: 043109.

[51] Hao F, Nordlander P. Efficient dielectric function for FDTD simulation of the optical properties of silver and gold nanoparticles. Chem Phys Lett 2007; 446: 115-8.

[52] Berini P, Charbonneau R, Lahoud N, Mattiussi G. Characterization of long-range surface-plasmon-polariton waveguides. J Appl Phys 2005; 98: 043109

[53] Ma YG, Li XY, Yu HK, Tong LM, Gu Y, Gong QH. Direct measurement of propagation losses in silver nanowires. Opt Lett 2010; 35: 1160-62.

[54] Vial A, Grimault A-S, Macías D, Barchiesi D, de la Chapelle ML. Improved analytical fit of gold dispersion: Application to the modeling of extinction spectra with a finite-difference time-domain method. Phys Rev B 2005; 71: 085416.

[55] Lynch DW, Hunter WR. In Handbook of optical constants of solids, edited by E. D. Palik (Academic, New York, 1985) 275-6. 